\title{
DURABILIDADE DA UNIÃO À CERÂMICA DE ZIRCÔNIA TETRAGONAL POLICRISTALINA CONTENDO ÍTRIO (Y-TZP)
}

\author{
Juliana Souza Carvalho Aluna do Curso de Odontologia da \\ Escola Bahiana de Medicina e Saúde \\ Pública \\ Marcelo Giannini Mestre e doutor, professor Adjunto da \\ Faculdade de Odontologia de \\ Piracicaba da Universidade de \\ Campinas \\ Andrea Nóbrega Cavalcanti \\ Mestre e doutora, professora Adjunto \\ do Curso de Odontologia e da \\ Faculdade de Odontologia da \\ Universidade Federal da Bahia
}

\begin{abstract}
Resumo
Objetivo: Este estudo avaliou in vitro a durabilidade da união do cimento resinoso à cerâmica Y-TZP, verificando o efeito de diferentes tratamentos de superfície e da aplicação prévia do sistema adesivo na peça. Metodologia: Quarenta placas de cerâmica foram distribuídas em quatro grupos experimentais (n=10) de acordo com a combinação entre tratamento de superfície (abrasão a ar ou irradiação com laser de Er:YAG) e aplicação de dois diferentes tipos de sistema adesivo (Scotchbond Multi Purpose - catalisador e Single Bond 2) sobre a peça previamente à inserção do cimento resinoso. Após o tratamento da cerâmica, cilindros de cimento resinoso foram construídos, utilizando matrizes com $0,8 \times 1 \mathrm{~mm}$ (diâmetro x altura). Em cada grupo experimental, 5 corpos-de-prova foram submetidos ao ensaio de microcisalhamento após $24 \mathrm{~h}$, e outros 5 corpos-de-prova foram testados após 6 meses em armazenamento em água. Resultados: Independentemente do adesivo e do tempo, as maiores médias foram obtidas nos grupos submetidos à abrasão a ar. Não foram detectadas diferenças significativas entre os sistemas adesivos e nem entre os tempos. Conclusão: Pode-se concluir que a abrasão a ar e o uso do agente adesivo quimicamente ativado sobre a peça podem melhorar a união à zircônia.
\end{abstract}

Palavras-chave: Cerâmica; Sistemas adesivos; Cimentação.

\section{DURABILITY OF THE BOND TO YTTRIA-STABILIZED ZIRCONIA CERAMIC (Y-TZP)}

\begin{abstract}
Purpose: This study evaluated in vitro the durability of the bond between resin cement and Y-TZP ceramic, testing the effect of different surface treatments and the previous application of an adhesive system over the ceramic. Methods: Forty Y-TZP ceramic plates were distributed into four experimental groups $(\mathrm{n}=10)$ according to the combination between surface treatment (air abrasion or Er:YAG laser irradiation) and adhesive system application (Scotchbond Multi Purpose - catalyst or Single Bond 2) in the plate previously to the use of the resin cement. After the surface treatment, resin cement cylinders were built using $0.8 \times 1 \mathrm{~mm}$ matrices (diameter $\mathrm{x}$ height). In each group, 5 specimens were tested for microshear after $24 \mathrm{~h}$, and other 5 specimens were tested after 6 months of water storage. Results: Regardless of the adhesive system or period, greater means were obtained in air abraded groups. No statistically significant difference between adhesive systems or periods. Conclusion: It could be concluded that the air abrasion improves bonding to T-TZP ceramic, regardless of the adhesive system used to coat zirconia ceramic surfaces.
\end{abstract}

Keywords: Ceramic; Adhesive systems; Luting. 


\section{INTRODUÇÃO}

A zircônia tetragonal policristalina estabilizada com ítrio (Y-TZP) vem sendo introduzida como uma nova classe de cerâmica odontológica; com reconhecimento advindo de bons resultados em pesquisas recentes. Embora a Y-TZP seja utilizada como biomaterial cerâmico em aplicações médicas desde o final dos anos 60, a sua utilização na odontologia é relativamente recente com maior impacto no campo da prótese e implantodontia.

As propriedades mecânicas de materiais de Y-TZP, tais como flexão e resistência à fratura, são consideravelmente superiores aos de outras cerâmicas dentais. ${ }^{(1,2)}$ Esta superioridade está relacionada ao mecanismo de transformação de fase induzida por tensão, semelhante ao explorado em aço temperado. ${ }^{(3)} \mathrm{O}$ óxido de zircônio $\left(\mathrm{ZrO}_{2}\right)$ é um material polimórfico que tem três alótropos: a fase monoclínica é estável até $11.708^{\circ} \mathrm{C}$ onde se transforma na fase tetragonal, que é estável até $23.708^{\circ} \mathrm{C}$; a fase cúbica existe até o ponto de fusão em $26.808^{\circ} \mathrm{C}$. A transformação da fase (t) tetragonal para a monoclínica (m) ocorre por um processo difusivo; gerando expansão volumétrica relativamente grande (3-5\%). Esta transformação pode ser induzida por uma tensão aplicada externamente, exercida por impacto ou fratura; e leva ao desenvolvimento de tensões internas que se opõem à abertura da fenda e, portanto, agem para evitar a propagação de trincas. ${ }^{(1,3,4)}$

Apesar ter melhores propriedades mecânicas, importantes para um desempenho de longo prazo de um material cerâmico, o sucesso clínico e longevidade das próteses fixas em cerâmica parece estar fortemente dependente do processo de cimentação e intimamente relacionado com a integridade do cimento na margem. ${ }^{(5)} \mathrm{O}$ baixo potencial adesivo é uma desvantagem das cerâmicas Y-TZP; pois existe um pensamento comum de que os métodos convencionais de cimentação adesiva, que incluem o condicionamento ácido prévio da superfície cerâmica com ácido fluorídrico mais silano, não são eficientes para a cerâmica YTZP, devido à sua falta de sílica e da fase vítrea. ${ }^{(6,7)}$ Por este motivo, estudos recentes têm sugerido técnicas de cimentação específicas para cerâmicas Y-TZP. Estas técnicas incluem métodos de tratamento da superfície, a exemplo do jateamento com óxido de alumínio, e da irradiação com laser de Er:YAG. ${ }^{(8)}$

O jateamento com partículas de óxido de alumínio é realizado rotineiramente para remover as camadas de contaminantes, aumentando assim a retenção micromecânica entre o cimento resinoso e a restauração. ${ }^{(5,9)}$ Geralmente, no jato usam-se partículas de óxido de alumínio com tamanhos variando entre $25 \mu \mathrm{m}$ a $250 \mu \mathrm{m}$. Estas partículas podem ou não ser 
revestido por sílica (tratamento triboquímico). ${ }^{(10)} \mathrm{O}$ efeito do jateamento sobre as propriedades mecânicas da cerâmica a base de zircônia tem sido reiteradamente discutidas na literatura; tanto resultados positivos como negativos tem sido descritos. ${ }^{(10-12)}$

O tratamento de superfície gera alterações microscópicas sobre a superfície, logo, o molhamento das superfícies tratadas é melhor realizado por agentes menos viscosos. Por este motivo, a aplicação de finas camadas de agentes de união sobre a peça cerâmica é constantemente indicada; porém, são limitadas as evidências científicas a respeito desta indicação. O objetivo do presente estudo foi avaliar in vitro o efeito de diferentes tratamentos de superfície (jateamento com partículas de óxido de alumínio e irradiação com laser de Er:YAG) e da aplicação prévia dos sistemas adesivos na superfície da peça, sobre a resistência de união do cimento resinoso à cerâmica Y-TZP, avaliada imediatamente ou após 6 meses de armazenamento em água.

\section{MATERIAIS E MÉTODOS}

Para este estudo, a cerâmica densa de zircônia do sistema Cercon Smart Ceramics (Degudent-Dentsply, Hanau, Alemanha) foi utilizada. Lingotes presinterizados foram seccionados em quarenta placas na dimensão de $5 \times 3 \times 0,75 \mathrm{~mm}$. Os espécimes foram incluídas em uma base de resina acrílica e limpas com álcool 70\%.

As placas de cerâmica foram aleatoriamente distribuídas em 2 grupos experimentais $(n=20)$, de acordo com o tratamento de superfície recebido (abrasão a ar ou a irradiação com laser). Os tratamentos de superfície estão descritos a seguir:

$\mathrm{Al}_{2} \mathrm{O}_{3}$ - A área da superfície da cerâmica a ser tratada foi delimitada com fita adesiva. As superfícies foram jateadas com partículas de $53 \mu \mathrm{m}$ de óxido de alumínio, sob pressão de 2,5 bar por 15 segundos a uma distância de $10 \mathrm{~mm}$. Após o jateamento, a fita adesiva foi removida e as placas foram limpas em banho de ultra-som durante 3 minutos.

Er:YAG - As superfícies cerâmicas foram irradiadas com laser de Er:YAG (erbium-doped yttrium aluminum garnet) por 5 segundos sob refrigeração constante com água. Previamente à irradiação com laser, as superfícies a serem irradiadas foram cobertas com uma camada de grafite para favorecer a absorção de energia e delimitada com fita adesiva. A intensidade de energia fora de $200 \mathrm{~mJ}$ e a taxa de repetição de $10 \mathrm{~Hz}$. Após a irradiação, a fita adesiva foi removida e as placas foram limpas conforme descrito no grupo anterior. 
Após o respectivo tratamento de superfície, as placas de cerâmica foram aleatoriamente divididas em 2 grupos experimentais $(n=10)$, segundo a aplicação do sistema adesivo:

SB2 - Foi aplicada uma fina camada do sistema adesivo Adper Single Bond 2 (3M-ESPE, Sumaré, São Paulo, Brazil) sobre a superfície interna da peça. A aplicação foi feita com microbrush embebido de adesivo e seu excesso removido com papel filtro. Houve fotoativação de acordo com as indicações do fabricante.

SBMP catalisador - Uma fina camada do catalisador do sistema adesivo Scotch Bond Multi Uso Plus (3M-ESPE, Sumaré, São Paulo, Brazil) foi aplicada sobre a superfície interna da peça com o auxilio de um microbrush e seu excesso foi removido com papel filtro.

Cilindros do cimento resinoso Rely-X ARC (3M-ESPE, Sumaré, São Paulo, Brazil) foram fixados sobre as placas imediatamente após a remoção do excesso do adesivo, utilizando matrizes com 1,33 x $1 \mathrm{~mm}$ (diametro x altura). A fotoativação do cimento foi realizada por 40 segundos. Após a fotoativação, as matrizes foram cortadas com lâmina de bisturi $\left(\mathrm{n}^{\circ} 15\right)$ e foram removidas.

Seguindo os procedimentos em todos os grupos experimentais, os corpos de prova foram aleatoriamente distribuídos em 2 grupos $(n=5)$, segundo o momento de avaliação da resistência de união: 24 horas após fotoativação do cimento ou após 6 meses de armazenamento. Durante o armazenamento, os corpos-de-prova foram mantidos em ambiente fechado e livre da luz, em água destilada a $37^{\circ} \mathrm{C}$; sendo a água trocada mensalmente.

Para avaliação da resistência de união, as placas de cerâmica foram fixadas no dispositivo de microcisalhamento; o ensaio foi realizado com velocidade de $0,5 \mathrm{~mm} / \mathrm{min}$, em maquina de ensaio universal (EMIC DL 10000, EMIC Equipamentos e Sistemas de Ensaio Ltda, São José dos Pinhais, Paraná, Brasil). Valores em KgF foram convertidos em MPa e tabulados para análise estatística. Os dados obtidos foram submetidos a uma análise exploratória para determinar adequação aos parâmetros da Análise de Variância (ANOVA). A análise estatística inferencial foi feita com a ANOVA a 3-critérios (tratamento $\mathrm{x}$ adesivo $\mathrm{x}$ tempo), incluindo todas as interações estatísticas no modelo e com nível de significância de $5 \%$.

Após o ensaio de resistência de união, as superfícies das placas de cerâmica foram avaliadas em microscopia óptica com 40x de aumento para classificar o padrão de fratura de acordo com a prevalência de uma das seguintes categorias: (A) falha adesiva ou parcialmente 
adesiva da interface entre cerâmica e cimento resinoso; $(\mathrm{Ce})$ falha coesiva da cerâmica; $(\mathrm{Cs})$ falha coesiva do sistema de cimentação.

\section{RESULTADOS}

A análise estatística não demonstrou interações significativas entre os fatores em estudo ( $p>0,05)$. Independentemente do adesivo e do tempo, diferenças significativas entre os níveis do fator tratamento foram observadas $(\mathrm{p}=0,002)$, e as maiores médias foram obtidas nos grupos submetidos à abrasão a ar. Não foram detectadas diferenças significativas entre os sistemas adesivos $(\mathrm{p}=0,36)$ e nem entre os tempos $(\mathrm{p}=0,63)$ (Tabela 1$)$.

Tabela 1 - Média (desvio-padrão) obtida nos grupos experimentais

\begin{tabular}{|c|c|c|c|c|c|}
\hline \multirow[t]{2}{*}{ Tempo } & \multirow[t]{2}{*}{ Tratamento } & \multicolumn{2}{|c|}{ Adesivo } & & \\
\hline & & SB2 & catalisador & & \\
\hline \multirow[t]{3}{*}{ Inicial } & Abrasão a ar & $2.0(1.5)$ & $2.9(1.9)$ & $\mathrm{A}$ & \\
\hline & Irradiação com laser & $1.1(0.6)$ & $1.2(0.6)$ & $\mathrm{B}$ & * \\
\hline & & $\mathrm{a}$ & $\mathrm{a}$ & & \\
\hline \multirow[t]{3}{*}{ 6-meses } & Abrasão a ar & $2.4(0.9)$ & $2.8(1.1)$ & A & \\
\hline & Irradiação com laser & $1.5(0,5)$ & $1.3(0.8)$ & $\mathrm{B}$ & * \\
\hline & & $\mathrm{a}$ & $\mathrm{a}$ & & \\
\hline
\end{tabular}

Letras distintas representam significância estatística (ANOVA 2-critérios, alfa=5\%). Maiúsculas comparam tratamentos e minúsculas comparam adesivos. Símbolos $(* / \wedge)$ representam diferenças entre tempos.

\section{DISCUSSÃO}

Os tratamentos de superfície investigados neste estudo resultaram em resistência de união à cerâmica Y-TZP significativamente diferentes. Um estudo anterior avaliou a topografia superficial de superfícies cerâmicas similares às do presente trabalho através de análises da topografia superficial e de imagens em microscopia eletrônica de varredura $(\mathrm{MEV}) .{ }^{(13)}$ As imagens deste estudo mostram que a abrasão do ar parece ser um método mais eficiente para modificar as superfícies da zircônia em comparação com a irradiação do laser. $^{(13)}$

A segunda hipótese investigada neste estudo foi de que tipos diferentes de sistema adesivo aplicados sobre as superfícies iriam resultar em diferenças na resistência de união. Inicialmente, foi proposto para o presente estudo a inclusão de um grupo controle, em que o 
sistema adesivo não seria utilizado. Porém, durante a realização da fase experimental, todos os corpos de prova deste grupo foram perdidos antes da avaliação da resistência de união, impedindo a sua avaliação.

De acordo com os resultados obtidos, não houve diferença significativa entre a resistência adesiva dos agentes adesivos utilizados, e ambos apresentaram maior força de adesão após abrasão a ar. Alguns estudos prévios também indicaram que a ligação superior a zircônia foi obtida quando as superfícies são abrasionadas a ar. ${ }^{(9,14)}$ Superfícies jateadas a ar podem apresentar uma área de superfície aumentada, o que favorece a molhamento pelos sistemas adesivos. No entanto, outros autores afirmam que as microporosidades criadas por tratamentos de superfície podem agir como iniciadores de fendas, enfraquecendo material cerâmico. ${ }^{(1)}$ Para uma melhor explicação a estas afirmações e negações futuros estudos devem ser realizados.

A irradiação da cerâmica Y-TZP com laser de Er: YAG vem sendo proposto como método de tratamento de superfície. ${ }^{(8)} \mathrm{O}$ laser de Er: YAG tem a capacidade de remover partículas por micro explosões e por evaporação, um processo chamado ablação. Durante o tratamento com laser, as mudanças de temperatura local devido ao aquecimento e esfriamento criam tensões internas que podem danificar a cerâmica. ${ }^{(13,15)}$ Estudos demonstraram o aumento da temperatura interfere nas propriedades mecânicas das cerâmicas Y-TZP pela indução da transformação de fase, ${ }^{(16)}$ o que indica a necessidade de uso de parâmetros de intensidade mais fracos para evitar estes efeitos. ${ }^{(13)}$ No presente estudo, quando o laser foi utilizado para tratar as superfícies, ambos os adesivos apresentaram resultados de resistência de união semelhantes. Porém, os dois sistemas adesivos apresentaram resistência de união inferior quando aplicados sobre superfícies jateadas, indicando que a irradiação a laser não foi tão eficaz em melhorar a resistência de união como a abrasão a ar.

Neste estudo, a resistência de união imediata (24 horas após a polimerização do cimento resinoso) e 6 meses após a polimerização do cimento resinoso sobre a peça foram testados. Uma vez que outros autores propõem que um período de exposição à água de apenas 24 horas é insuficiente para permitir a difusão de água na interface adesiva. ${ }^{(8)}$ Porém, os resultados não demonstraram diferenças significativas entre os tempos experimentais, demonstrando que a união permaneceu estável ao longo dos seis meses de armazenamento. 


\section{CONCLUSÃO}

Independentemente do sistema adesivo utilizado, o jateamento das superfícies de zircônia com óxido de alumínio apresentou maior resistência de união comparada à irradiação com laser de Er:YAG. No entanto, ambos os tratamentos e sistemas adesivos foram capazes de manter a adesão após seis meses de armazenamento em água.

\section{REFERÊNCIAS}

1. Piconi C, Maccauro G. Zirconia as a ceramic biomaterial. Biomaterials. 1999;20:1-25.

2. Luthy H, Loeffel O, Hammerle $\mathrm{CH}$. Effect of thermocycling on bond strength of luting cements to zirconia ceramic. Dent Mater. 2006;22:95-200.

3. Anusavice KJ. Phillips's science of dental materials. St Louis: Elsevier Science; 2003.

4. Kosmac T, Oblak C, Jevnikar P, Funduk N, Marion L. The effect of surface grinding and sandblasting on flexural strength and reliability of Y-TZP zirconia ceramic. Dent Mater. 1999; 15:426-433.

5. Valandro LF, Ozcan M, Bottino MC, Bottino MA, Scotti R, Bona AD. Bond strength of a resin cement to high-alumina and zirconia-reinforced ceramics: The effect of surface conditioning. J Adhes Dent. 2006;8:175-181.

6. Derand T, Molin M, Kvam K. Bond strength of composite luting cement to zirconia ceramic surfaces. Dent Mater. 2005;21:1158-62.

7. Kern M, Wegner SM. Bonding to zirconia ceramic: adhesion methods and their durability. Dent Mater. 1998;14:64-71.

8. Cavalcanti AN, Foxton RM, Watson TF, Oliveira MT, Giannini M, Marchi GM. Bond strength of resin cements to a zirconia ceramic with different surface treatments. Oper Dent. 2009;34:268-275.

9. Wolfart M, Lehmann F, Wolfart S, Kern M. Durability of the resin bond strength to zirconia ceramic after using different surface conditioning methods. Dent Mater. 2007;23:4550 .

10. Curtis AR, Wright AJ, Fleming GJ. The influence of surface modification techniques on the performance of a YTZP dental ceramic. J Dent. 2006;34:195-206.

11. Zhang Y, Lawn BR, Rekow ED, Thompson VP. Effect of sandblasting on the long-term performance of dental ceramics. J Biomed Mater Res B Appl Biomater. 2004;71:381-386. 
12. Papanagiotou HP, Morgano SM, Giordano RA, Pober R. In vitro evaluation of lowtemperature aging effects and finishing procedures on the flexural strength and structural stability of Y-TZP dental ceramics. J Prosthet Dent. 2006;96:154-164.

13. Cavalcanti AN, Pilecki P, Foxton RM, Watson TF, Oliveira MT, Giannini M et al. Evaluation of the surface roughness and morphologic features of Y-TZP ceramics after different surface treatments. Photomed Laser Surg. 2009;27:473-9.

14. Ozcan M, Kerkdijk S, Valandro LF. Comparison of resin cement adhesion to Y-TZP ceramic following manufacturers' instructions of the cements only. Clin Oral Investig. 2007;13:279-282.

15. Gokce B, Ozpinar B, Dundar M, Comlekoglu E, Sen BH, Gungor MA. Bond strengths of all-ceramics: Acid vs laser etching. Oper Dent. 2007;32:173-178.

16. Guazzato M, Quach L, Albakry M, Swain MV. Influence of surface and heat treatments on the flexural strength of Y-TZP dental ceramic. J Dent 2005;33:9-18. 